\title{
Diseño de estrategias de optimización del volumen de reinfusión en hemodiafiltración en línea
}

\author{
Ana Ciriza Aramburu \\ Inmaculada Arranz Pérez \\ María Adelaida Martín Carrasco
}

\author{
Clínica San Carlos \\ Nacional Medical Care of \\ Spain S.A. Sevilla
}

\section{RESUMEN}

El diseño de algoritmos clínicos para la optimización del volumen de reinfusión en Hemodiafiltración en línea en 16 pacientes con insuficiencia renal crónica ha permitido incrementar este parámetro relacionado con la eficacia de la técnica, creando un lenguaje intermedio entre los diferentes especialistas que intervienen en el problema. Una de las consecuencias más importantes ha sido la disminución de la variabilidad de la práctica clínica y de enfermería a la hora de establecer en estos pacientes el $\mathrm{Q}_{\mathrm{I}}$ óptimo. A consecuencia de estas estrategias, los resultados preliminares se ajustan al objetivo propuesto.

PALABRAS CLAVE: HEMODIAFILTRACIÓN EN LÍNEA VOLUMEN DE REINFUSIÓN HEMOCONCENTRACIÓN PERFIL DE ULTRAFILTRACIÓN

\section{DESIGN OF STRATEGIES TO OPTIMIZE THE REINFUSION VOLUME IN ONLINE HAEMODIA- FILTRATION}

\section{ABSTRACT}

The design of clinical algorithms to optimize the volume of reinfusion in online haemodiafiltration in 16

$$
\begin{gathered}
\text { Correspondencia: } \\
\text { Ana Ciriza Aramburu } \\
\text { Clínica San Carlos } \\
\text { Nacional Medical Care of Spain S.A. } \\
\text { c/ Marqués Luca de Tena } 16 \\
\text { 41013 Sevilla }
\end{gathered}
$$

patients with chronic renal insufficiency has made it possible to increase this parameter related to the efficacy of the technique, creating an intermediate language among the different specialists involved in the problem. One of the most important consequences has been to reduce the variability of clinical and nursing practice in establishing the optimum QI in such patients. As a result of these strategies, the preliminary results respond to the proposed aim.

KEY WORDS: $\quad$ ONLINE HAEMODIAFILTRATION

REINFUSION VOLUME

HAEMOCONCENTRATION

ULTRAFILTRATION PROFILE

\section{INTRODUCCIÓN}

La Hemodiafiltración (Hdf) en línea se ha mostrado como una técnica de tratamiento de la insuficiencia renal crónica (IRC) muy efectiva para la eliminación de las moléculas de mediano y pequeño tamaño. Además la estabilidad cardiovascular que proporciona la señala como el tratamiento de elección en pacientes con inestabilidad cardiovascular ${ }^{(1)}$. La Hdf en línea utiliza mejor la permeabilidad de las membranas sintéticas forzando la ultrafiltración dentro de los márgenes de seguridad del paciente y de integridad del sistema, consiguiendo una eliminación mayor de solutos de pequeño y mediano peso molecular, algunos de ellos patológicos e indicadores de las complicaciones más frecuentes a largo plazo como amiloidosis, enfermedades cardiovasculares, inflamación y desnutrición. La producción en linea de una cantidad ilimitada de solución dializadora estéril a bajo 
coste ha favorecido su empleo cada vez más frecuente. Está descrita una mejor tolerancia a la Hdf en línea frente a las otras técnicas. Nosotros hemos comparado algunos parámetros relativos a la calidad de vida de estos pacientes y hemos comprobado una mejoría significativa del estado nutricional (peso corporal) sobre todo en pacientes con edades inferiores a los 55 años, probablemente secundaria a la mejora del apetito y de la ingesta proteica, con un incremento del IMC de 22,9 a $25,5^{(2)}$

La mejoría de los parámetros bioquímicos y de inflamación tisular hace que se consigan mayores elevaciones del Hematocrito (Hcto) con menores dosis de Eritropoyetina si se compara con otras técnicas de diálisis ${ }^{(3,4)}$. Precisamente esta elevación de Hcto es una de las razones por las que se puede limitar la eficacia de la técnica, ya que en el caso de la Hdf en línea con post-dilución, el aumento de la Presión Transmembrana (PTM) puede producir una Hemoconcentración (HC). La PTM está relacionada con el Hcto, el flujo sanguíneo $\left(Q_{B}\right)$, el volumen de reinfusión $\left(\mathrm{Q}_{\mathrm{J}}\right)$, la ultrafiltración horaria (Ufh) y el coeficiente de ultrafiltración (Kuf) del dializador. La $\mathrm{HC}$ no se produce con la Hdf en línea con técnica predilucional, si bien ésta es menos efectiva que la post-dilucional ${ }^{(5)}$, también se minimizaría con la técnica mixta pre/postdilucional ${ }^{(6)}$.

Ya que la mayor eficacia de la técnica está directamente relacionada con el $\mathrm{Q}_{\mathrm{I}}$ empleado $^{(7,8)}$, todos los esfuerzos se centran en elevar este parámetro. El aumento del $\mathrm{Q}_{\mathrm{B}}$ y dializadores con mayores KUf que bajan la PTM, son las principales variables sobre las que se puede actuar. La Ufh está directamente relacionada con la PTM, ésta va subiendo progresivamente de forma que las posibilidades de que se produzca HC aumentan a medida que trascurre la sesión de tratamiento.

\section{OBJETIVO}

El objetivo de este trabajo es presentar el diseño de las estrategias de optimización del $\mathrm{Q}_{\mathrm{I}}$ en una serie de pacientes con IRC tratados en un Centro de diálisis.

\section{MATERIAL Y MÉTODOS}

Se plantea un estudio prospectivo, tipo caso-control. De los 53 pacientes que actualmente se dializan en nuestra
Unidad con Hdf en línea, se seleccionaron 22 en los que la Ufh fuese más constante a lo largo de la semana en los 3 meses anteriores al estudio o que por su situación cardiovascular, se intuyera que pudieran soportar Ufh importantes en determinados momentos de la sesión de Hdf en línea, cuando se aplicara un perfil de ultrafiltración.

Todos los pacientes se dializan en monitores Fresenius 4008S y dializadores con membranas de helixona de $1,4 \mathrm{mts}^{2}$ y Kuf de $46 \mathrm{ml} / \mathrm{h}$ *mmHg (FX60) salvo 1 caso con membrana de polisulfona de 1,8 mts2 y Kuf de 55 $\mathrm{ml} / \mathrm{h}$ *mmHg (HF80S). Las agujas de punción se personalizan para cada paciente con calibres $14 \mathrm{G}$ y $15 \mathrm{G}$.

Se determinaron Hcto a todos los pacientes al inicio y en las semanas pares del estudio, presión arterial (PA), venosa (PV) y transmembrana (PTM), al inicio de la sesión, a los 60', 120', 180', 210' y final de la sesión; así mismo se anotaron las HC y estado de coagulación de los dializadores y líneas de sangre. Estos datos fueron recogidos de forma prospectiva en una hoja de cálculo Excel de Office.

En la figura 1 se describe el algoritmo de decisión utilizado en la estrategia de optimización del $\mathrm{Q}_{\mathrm{T}}$. A cada paciente se le prescribe un $Q_{B}$ fijo durante todo el estudio medido según el $\mathrm{Q}_{\mathrm{B}}$ efectivo que muestra el Monitor de Hdf en línea. La Ufh se mantiene constante para cada día de la semana. El $\mathrm{Q}_{\mathrm{I}}$ se establece partiendo del que históricamente se había prescrito y que no producía HC. A ese valor se le aumenta un 5\% si el Hcto es superior o igual a $40 \%$ y un $10 \%$ si éste es inferior. $\mathrm{Si}$ transcurrida una semana el paciente no presenta más de una $\mathrm{HC}$, se repite el aumento de $\mathrm{Q}_{\mathrm{I}}$ hasta la aparición de dos $\mathrm{HC}$ en una semana. $\mathrm{El}_{1}$ que tenga prescrito en ese momento, se toma entonces como valor de referencia para aplicar durante una semana un perfil de Ufh descendente y constante para el valor total de pérdida de peso programada. Si el paciente presenta alguna HC terminará esta fase del estudio, en caso contrario se continua el mismo incremento del $\mathrm{Q}_{\mathrm{I}}$ en un $5 \%$ sobre el valor anterior, independientemente del Hcto del paciente, repitiendo este esquema hasta la aparición de una $\mathrm{HC}$ que suponga la finalización del estudio. Se define por fin el $\mathrm{Q}_{\mathrm{I}}$ óptimo como el resultante de la media entre los 2 últimos valores utilizados.

A todos los pacientes seleccionados, se les pidió y obtuvo consentimiento informado. 


\section{RESULTADOS}

Presentamos los resultados preliminares del trabajo realizado hasta este momento. De los 22 pacientes que cumplían los requisitos de inclusión, siguen en el estudio 16. Un paciente abandonó el estudio por trasplante renal, otro por problemas con el acceso vascular, otro por traslado temporal a otra Unidad de Diálisis y tres por deseo de los pacientes de no continuar en el protocolo antes de terminar la primera semana del estudio.

El algoritmo fue diseñado de forma interactiva al incorporar diferentes estrategias a la vista de los resultados obtenidos. En la figura 1 se representa la versión actual del algoritmo.

En las figuras 2 y 3 se representa con código de colores las vías seguidas por 2 pacientes. Comprobamos un incremento significativo del $\mathrm{Q}_{\mathrm{I}}$ que oscila entre un 12,92 y un $34,95 \%$ del valor inicial, cifras que consideramos muy significativas.

En la tabla 1 se muestran los resultados obtenidos en los pacientes que han finalizado el estudio. Oscilan entre un 10 y un $34,95 \%$. Como se observa en 2 pacientes no se ha conseguido aumentar el $\mathrm{Q}_{\mathrm{I}}$ en la fase del estudio en la que se aplica el perfil de ultrafiltración, en uno de ellos se explica por incremento excesivo del Hcto, sin hallar razones para el otro caso.

\section{DISCUSIÓN}

La prescripción del $\mathrm{Q}_{\mathrm{I}}$ es personalizada y muy arbitraria, las diferentes fórmulas propuestas, basadas en el $Q_{B}\left(Q_{I}\right.$ $=\mathrm{QB}) \mathrm{o}$ en los diferentes aspectos que inciden como Hcto, $Q_{B}$, Kuf del dializador y proteínas totales no son útiles para la práctica diaria. La metodología utilizada en este estudio para la optimización del $\mathrm{Q}_{\mathrm{I}}$, con el diseño interactivo de un algoritmo, es original. Entre sus ventajas está la de constituir un lenguaje intermedio entre los facultativos, el personal de enfermería y los metodólogos que permite diseñar estrategias o vías clínicas dirigidas a conseguir los objetivos previstos.
Los resultados preliminares permiten comprobar como este tipo de herramientas facilitan el seguimiento del proceso de optimización en un paciente individual o en un grupo de pacientes con características similares.

\section{CONCLUSIONES}

Creemos que este objetivo ha sido conseguido con creces, como se comprueba en los resultados preliminares que demuestran un incremento significativo de la variable estudiada.

\section{BIBLIOGRAFÍA}

1. Canaud B, Bosc JY, Leray H, Stec F, Argiles A, Leblanc $\mathrm{M}$ et alt. On-line hemodiafiltration: state of the art. Nephrol Dial Transplant 1998; 13 (sup5):3-11

2. Castañeda Cano C, Ciriza Aramburu A, Díez Cano R. Hemodiafiltración en línea (HDF) en 52 pacientes: Evolución clínica y analítica. Rev Soc Esp Enferm Nefrol 2005; 8 (3): 12-15

3. Bonforte G, Beretta M, Baj A, Dozio B, Orlandini G, Marcelli $D$ et al. Anemia and hemodiafiltration with on-line production of infusion fluid. Nephrol Dial Transplant 1997; 12:A168

4. Maduell F, Del Pozo C, García H, Sánchez I, Hernández Jaras J, Albero MD et al. Cambio de hemodiafiltración convencional a hemodiafiltración en línea. Seguimiento de 1 año. Nefrología 1998; 18:308-315

5. Arenholz P, Winkler RE, Ramlow W, Tiess M, Müller $\mathrm{W}$. On-line hemodiafiltration with pre and post dilution: a comparison of efficaci. Int $J$ Artif Organs 1997; 20: 81-90

6. Pedrini LA, De Cristofaro V, Pagliari B, Sama F. Mixed predilution and postdilution online hemodiafiltration compared with the traditional infusion modes. Kidney Int 2000; 58(5): 2155-65

7. Lornoy W, Becaus I, Billiouw JM, Sierens L, van Malderen P. Remarkable removal of beta-2-microglobulin by on-line hemodiafiltration. Am J Nephrol 1998; 18:105-08

8. Wizemann V, Külz M, Techert F, Nederlof B. Efficacy of haemodiafiltration. Nephrol Dial Transplant 2001; 16 (sup 4): 27-30 


\begin{tabular}{|c|c|c|c|c|c|c|c|c|}
\hline Paciente & $Q_{1}$ Histórico & $\begin{array}{l}\text { Q, Máx sin } \\
\text { perfil ni HC }\end{array}$ & $\begin{array}{l}\text { Incremento } \\
\% \text { sin perfil }\end{array}$ & $\begin{array}{l}Q_{1} \text { inicial } \\
\text { con perfil }\end{array}$ & $\begin{array}{c}Q_{1} \text { con } \\
\text { perfil y HC }\end{array}$ & $Q_{1}$ óptimo & $\begin{array}{c}\text { Incremento } \\
\% \\
\text { conseguido } \\
\text { con el } \\
\text { perfil }\end{array}$ & $\begin{array}{c}\text { Incremento } \\
\% \text { total }\end{array}$ \\
\hline A.D.L. & 90 & 95 & 5,56 & 100 & 105 & 102 & 7,37 & 12,92 \\
\hline J.M.B. & 90 & 110 & 22,22 & 121 & 127 & 124 & 12,73 & 34,95 \\
\hline P.M.F. & 90 & 90 & 0,00 & 95 & 104 & 102 & 13,33 & 13,33 \\
\hline J.M.J.L. & 80 & 88 & 10,00 & 96 & 96 & 88 & 0,00 & 10,00 \\
\hline M.M.M & 80 & 92 & 15,00 & 101 & 110 & 105 & 14,13 & 29,13 \\
\hline J.E.C. & 80 & 97 & 21,25 & 103 & 103 & 97 & 0,00 & 21,25 \\
\hline
\end{tabular}

J.M.J.L. Hcto $>48 \%$

Tabla 1. Resultados obtenidos en los pacientes que han finalizado el estudio.



Figura 1: Algoritmo de decisión utilizado en la estrategia de optimización del $\mathrm{Q}_{\mathrm{I}}$ 


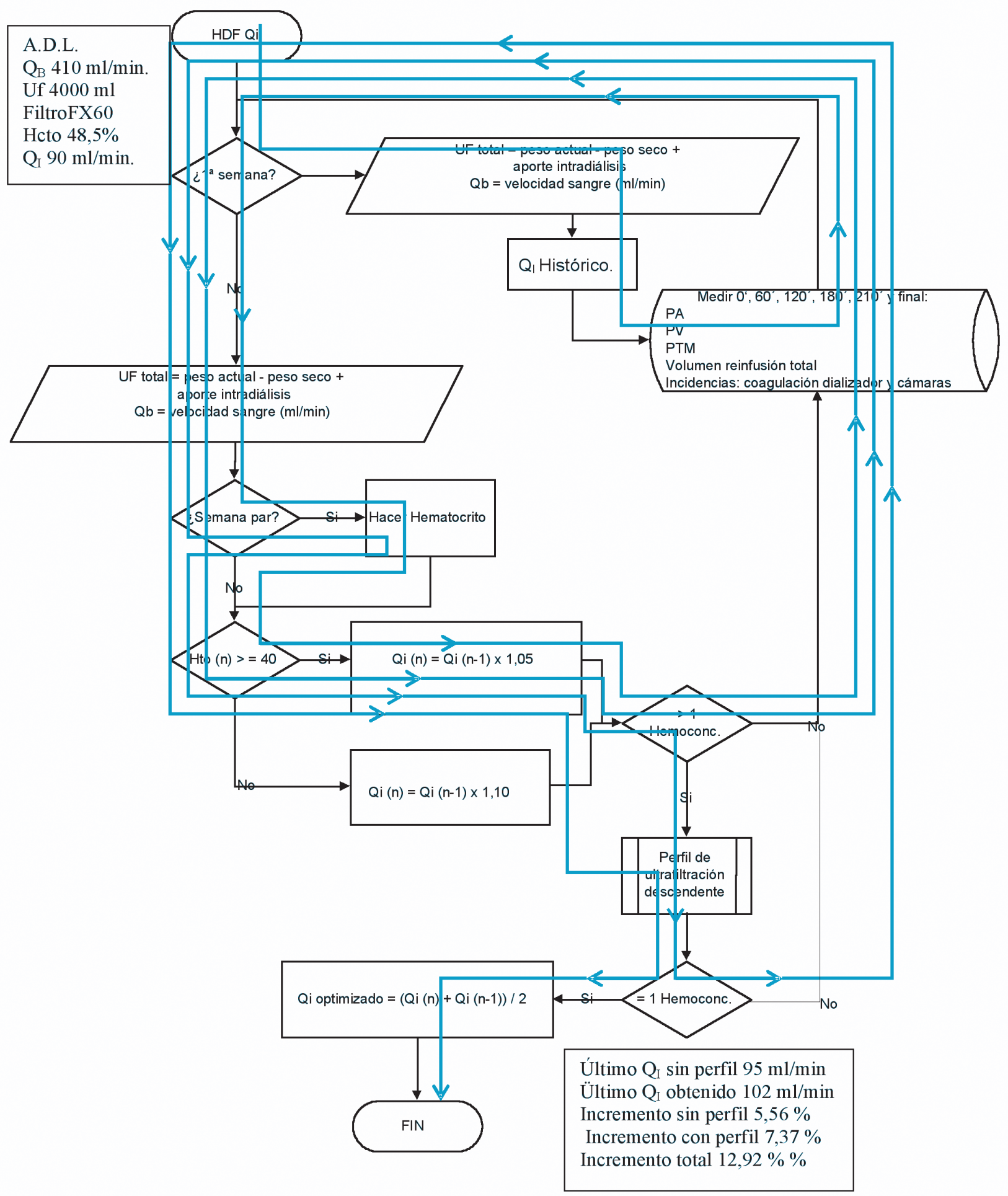

Figura 2. Vía seguida por un paciente con hematocritos superiores al 40\% durante el proceso de optimización de su $\mathrm{Q}_{\mathrm{I}}$, incrementos semanales del 5\%, hasta alcanzar un incremento total del $12,92 \%$ 


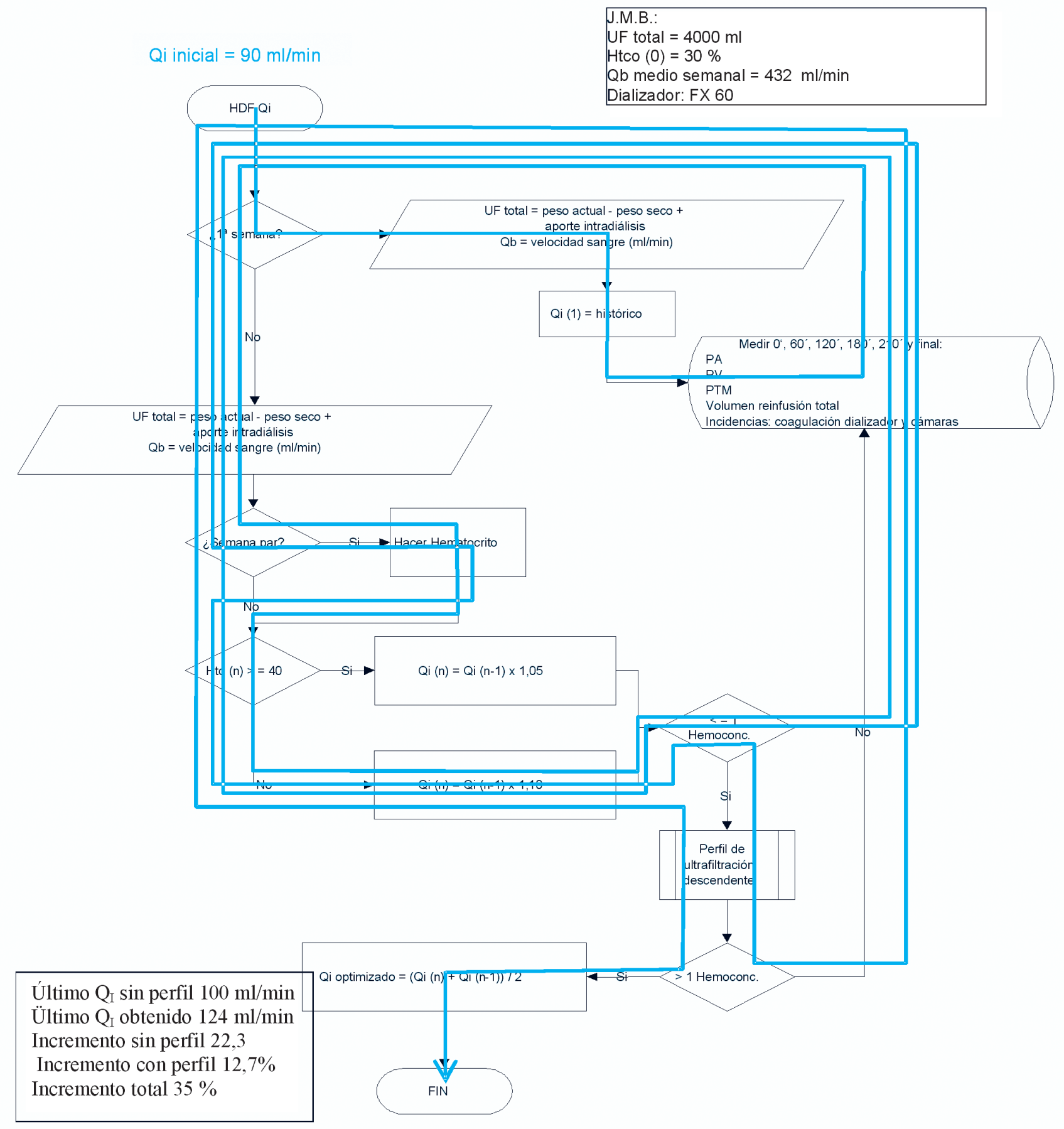

Figura 3. Vía seguida por un paciente con hematocritos inferiores al $40 \%$ durante el proceso de optimización de su $Q_{\Gamma}$. Los incrementos semanales del 10 $\%$ y la utilización del perfil permitieron alcanzar un incremento del $\mathrm{Q}_{\mathrm{I}}$ superior en un $35 \%$ al inicial. 